\title{
Asymptotic Expansions of Feynman Integrals on the Mass Shell in Momenta and Masses
}

\author{
V.A. Smirnov* \\ * Nuclear Physics Institute of Moscow State University \\ Moscow 119899, Russia
}

\begin{abstract}
A brief review of recent results on asymptotic expansions of Feynman integrals on the mass shell in momenta and masses and their application to 2-loop calculations is presented.
\end{abstract}

\section{INTRODUCTION}

Explicit formulae for asymptotic expansions of Feynman diagrams in various limits of momenta and masses, with the large off-shell momenta, have been derived in the simplest form in [1-3] - see a review, from the point of view of 1995, in ref. [4]. (See also informal arguments in [5].)

In this note, we present a brief review of recent results obtained for two typical limits with the large momenta on the mass shell: the limit of the large momenta on the mass shell with the large mass (in Section 2) and the Sudakov limit, with the large momenta on the massless mass shell (in Section 3). In particular, the presented explicit formulae provide coefficients at all powers and logarithms for two-loop diagrams in the Sudakov limit.

\section{LARGE MOMENTUM EXPANSION ON THE MASS SHELL}

Let us consider asymptotic expansion of a Feynman diagram $F_{\Gamma}(\underline{Q}, \underline{q}, \underline{M}, \underline{m})$ corresponding to a graph $\Gamma$ in the limit when the momenta $Q \equiv$ $\left\{Q_{1}, \ldots Q_{i}, \ldots\right\}$ and the masses $\underline{M} \equiv\left\{M_{1}, \ldots M_{i}, \ldots\right\}$ are larger than $\underline{q} \equiv$ $\left\{q_{1}, \ldots q_{i}, \ldots\right\}$ and $\underline{m} \equiv\left\{m_{1}, \ldots m_{i}, \ldots\right\}$. We suppose that the external momenta are non-exceptional and that $\left(\sum_{i \in I} Q_{i}\right)^{2} \neq M_{j}^{2}$, for any subset of indices $I$. Moreover, let the large external momenta be on the mass shell, $Q_{j}^{2}=M_{j}^{2}$.

(C) 1995 American Institute of Physics 
To obtain explicit formulae of the corresponding asymptotic expansion, a method [6,7] based on constructing a remainder of the expansion, written through an appropriate $R$-operation and using then diagrammatic Zimmermann identities have been applied in refs. $[8,9]$. The resulting explicit formula looks like

$$
F_{\Gamma}(\underline{Q}, \underline{M}, \underline{q}, \underline{m} ; \epsilon) \stackrel{M_{j}}{\sim} \underset{\sim}{\sim} \sum_{\gamma} \mathcal{M}_{\gamma} F_{\Gamma}(\underline{Q}, \underline{M}, \underline{q}, \underline{m} ; \epsilon)
$$

The operator $\mathcal{M}_{\gamma}$ happens to be a product $\prod_{i} \mathcal{M}_{\gamma_{i}}$ of operators of Taylor expansion in certain momenta and masses. For connectivity components $\gamma_{i}$ other than $\gamma_{0}$ (this is the component with the large external momenta), the corresponding operator performs Taylor expansion of the Feynman integrand $F_{\gamma_{i}}$ in its small masses and external momenta. The component $\gamma_{0}$ can be naturally represented as a union of its 1PI components and cut lines (after a cut line is removed the subgraph becomes disconnected; here they are of course lines with the large masses). By definition $\mathcal{M}_{\gamma_{0}}$ is again factorized and the Taylor expansion of the 1PI components of $\gamma_{0}$ is performed as in the case of c-components $\gamma_{i}, i=1,2, \ldots$.

It suffices now to describe the action of the operator $\mathcal{M}$ on the cut lines. Let $l$ be such a line, with a large mass $M_{j}$, and let its momentum be $P_{l}+k_{l}$ where $P_{l}$ is a linear combination of the large external momenta and $k_{l}$ is a linear combination of the loop momenta and small external momenta. If $P_{l}=Q_{i}$ then the operator $\mathcal{M}$ for this component of $\gamma$ is $\left.\mathcal{T}_{\kappa} \frac{1}{\kappa k_{l}^{2}+2 Q_{i} k_{l}}\right|_{\kappa=1}$. (We use $\mathcal{T}_{\ldots}$. to denote the operator of Taylor expansion at zero values of the corresponding variables.) In all other cases, e.g. when $P_{l}=0$, or it is a sum of two or more external momenta, the Taylor operator $\mathcal{M}$ reduces to ordinary Taylor expansion in small (with respect to this line considered as a subgraph) external momenta, i.e. $\left.\mathcal{T}_{\kappa} \frac{1}{\left(\kappa k_{l}+P_{l}\right)^{2}-M_{i}^{2}}\right|_{\kappa=1}$. Note that in all cases apart from the cut lines with $P_{l}^{2}=M_{j}^{2}$ the action of the corresponding operator $\mathcal{M}$ is graphically described (as for the off-shell limit) by contraction of the corresponding subgraph to a point and insertion of the resulting polynomial into the reduced vertex of the reduced graph.

The formula (1) was illustrated in [8] through a one-loop example and applied in [9] to calculation of the master two-loop diagram shown in Fig. 1a (where thick (thin) lines correspond to the mass $M(m)$ and the external momentum is at $\left.Q^{2}=M^{2}\right)$. There are four subgraphs, Fig. 1(a-d), that contribute to the general formula. For example, the action of the operator $\mathcal{M}_{\gamma}$ for $\gamma=$ Fig. 1d on the given Feynman integral

$$
\iint \frac{\mathrm{d}^{d} k \mathrm{~d}^{d} l}{\left(k^{2}-m^{2}\right)\left(l^{2}-m^{2}\right)\left(k^{2}+2 Q k\right)\left[(k+l)^{2}+2 Q(k+l)\right]\left(l^{2}+2 Q l\right)} .
$$




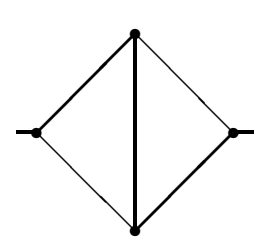

(a)

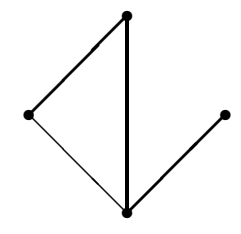

(b)

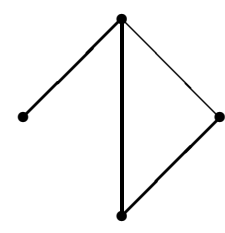

(c)

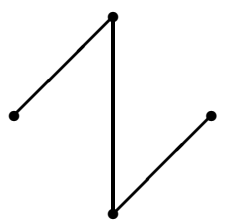

(d)

FIGURE 1. (a) Two-loop master self-energy diagram. (a-d) The subgraphs contributing to its large mass expansions (1) at $q^{2}=M^{2}$.

reduces to Taylor expansion of the last three factors in the integrand, resp., in $k^{2},(k+l)^{2}$, and $l^{2}$. Each term of the corresponding asymptotic expansion happens to be analytically computable - see details and results in [9].

Note that similar results for a simpler propagator-type 2-loop diagram in the same limit were obtained and applied in ref. [10]. Further applications of the presented explicit asymptotic expansions can be found in refs. [11].

\section{ASYMPTOTIC EXPANSION IN THE SUDAKOV LIMIT}

One of possible versions of the Sudakov limit is formulated as the behaviour of a three-point Feynman diagram $F_{\Gamma}\left(p_{1}, p_{2}, m\right)$ depending on two momenta, $p_{1}$ and $p_{2}$, on the massless mass shell, $p_{i}^{2}=0$, with $q^{2} \equiv-Q^{2}=\left(p_{1}-p_{2}\right)^{2} \rightarrow$ $-\infty$. We suppose, for simplicity, that there is one small non-zero mass, $m$.

To derive the simplest explicit formula for this limit, the previous strategy of constructing a remainder determined by an appropriate pre-subtraction operator and then applying Zimmermann identities can be used. Eventually, it takes an explicit form [8] similar to (1), with another operator $\mathcal{M}_{\gamma}$ :

$$
F_{\Gamma}\left(p_{1}, p_{2}, m, \epsilon\right) \stackrel{q^{2}}{\rightarrow^{\sim}} \sum_{\gamma} \mathcal{M}_{\gamma} F_{\Gamma}\left(p_{1}, p_{2}, m, \epsilon\right) .
$$

Here the sum runs over subgraphs $\gamma$ of $\Gamma$ for which at least one of the following conditions holds:

(i) In $\gamma$ there is a path between the end-points 1 and 3. (The end-points of the diagram are numerated according to the following order: $p_{1}, p_{2}, q=$ $p_{1}-p_{2}$.) The graph $\hat{\gamma}$ obtained from $\gamma$ by identifying these end-points is 1PI.

(ii) Similar condition with $1 \leftrightarrow 2$.

The pre-subtraction operator $\mathcal{M}_{\gamma}$ is now defined as a product $\prod_{j} \mathcal{M}_{\gamma_{j}}$ of operators of Taylor expansion acting on 1PI components and cut lines of the subgraph $\gamma$. Suppose that the above condition (i) holds and (ii) does not hold. Let $\gamma_{j}$ be a 1PI component of $\gamma$ and let $p_{1}+k$ be one of its external momenta, where $k$ is a linear combination of the loop momenta. (We imply that the loop 
momenta are chosen in such a way that $p_{1}$ flows through all $\gamma_{j}$ and the corresponding cut lines). Let now $\underline{q}_{j}$ be other independent external momenta of $\gamma_{j}$. Then the operator $\mathcal{M}$ for this component is defined as $\mathcal{T}_{k-\left(\left(p_{1} k\right) /\left(p_{1} p_{2}\right)\right) p_{2}, \underline{q}_{j}, \underline{m}_{j}}$, where $\underline{m}_{j}$ are the masses of $\gamma_{j}$. In other words, it is the operator of Taylor expansion in $\underline{q}_{j}$ and $\underline{m}_{j}$ at the origin and in $k$ at the point $\tilde{k}=\frac{\left(p_{1} k\right)}{\left(p_{1} p_{2}\right)} p_{2}$ (which depends on $k$ itself).

For the cut lines the same prescription is adopted. If $p_{1}+k$ is the momentum of the cut line, then the corresponding operator acts as $\left.\mathcal{T}_{\kappa} \frac{1}{\kappa\left(k_{l}^{2}-m_{l}^{2}\right)+2 p_{1} k}\right|_{\kappa=1}$. If both $(i)$ and $(i i)$ hold the corresponding operator performs Taylor expansion in the mass and the external momenta of subgraphs (apart from $p_{1}$ and $p_{2}$ ).

The formula (3) was illustrated in [8] through a one-loop example and applied in [12] to calculation of the diagram shown in Fig. 2a in two cases: (a) $m_{1}=\ldots=m_{5}=0, m_{6}=m$, and (b) $m_{1}=\ldots=m_{4}=0, m_{5}=m_{6}=m$.

For example, in the latter case of the Feynman integral

$$
\begin{aligned}
F_{i}\left(p_{1}, p_{2}, m, \epsilon\right) & =\iint \frac{\mathrm{d}^{d} k \mathrm{~d}^{d} l}{\left(k^{2}-2 p_{1} k\right)\left(k^{2}-2 p_{2} k\right)\left(k^{2}-m^{2}\right)} \\
& \times \frac{1}{\left(l^{2}-2 p_{1} l\right)\left(l^{2}-2 p_{2} l\right)\left((k-l)^{2}-m^{2}\right)},
\end{aligned}
$$

the subgraphs that give non-zero contributions to the general formula (3) are shown in Fig. 2a-f. The most complicated contribution comes from Fig. 2e and $\mathrm{f}$, when the corresponding subtraction operator expand propagators $1 /\left(\left(k^{2}-2 p_{1} k\right)\left(l^{2}-2 p_{1} l\right)\right)$ (respectively, $\left.1 /\left(\left(k^{2}-2 p_{2} k\right)\left(l^{2}-2 p_{2} l\right)\right)\right)$ in Taylor series in $k^{2}$ and $l^{2}$. In ref. [12], all the terms at the leading power were analytically calculated, with the aid of Mellin-Barnes and $\alpha$-parametric representations. Using the method of integration by parts within dimensional regularization [13] one can arrive at recurrence relations that provide all the relevant integrals contributing to an arbitrary power of the expansion. For example, here are results for the first four powers in the expansion of this diagram:

$$
\begin{array}{r}
-\frac{1}{\pi^{4}}\left(Q^{2}\right)^{2} F_{\Gamma}\left(Q^{2}, m^{2}\right) \stackrel{Q^{2}}{\sim} \sim \infty \frac{1}{24} \ln ^{4} t+\frac{\pi^{2}}{3} \ln ^{2} t-6 \zeta(3) \ln t+\frac{31}{2} \zeta(4) \\
-\frac{1}{t}\left(\frac{1}{6} \ln ^{3} t-\frac{3}{2} \ln ^{2} t+6 \ln t+\frac{2 \pi^{2}}{3} \ln t+18-\frac{\pi^{2}}{3}-6 \zeta(3)\right) \\
-\frac{1}{t^{2}}\left(\frac{1}{12} \ln ^{3} t-\frac{35}{8} \ln ^{2} t+\frac{29}{4} \ln t+\frac{\pi^{2}}{3} \ln t+\frac{153}{8}-\frac{19 \pi^{2}}{12}-3 \zeta(3)\right) \\
-\frac{1}{t^{3}}\left(\frac{1}{18} \ln ^{3} t-\frac{55}{6} \ln ^{2} t+\frac{503}{36} \ln t+\frac{2 \pi^{2}}{9} \ln t+\frac{1061}{36}-\frac{355 \pi^{2}}{108}-2 \zeta(3)\right),
\end{array}
$$

where $t=Q^{2} / m^{2}$. 


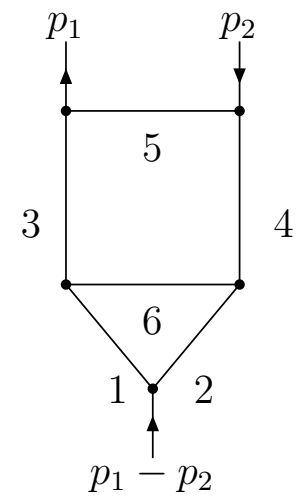

(a)
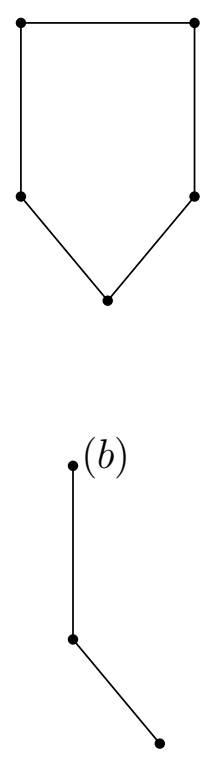

$(e)$
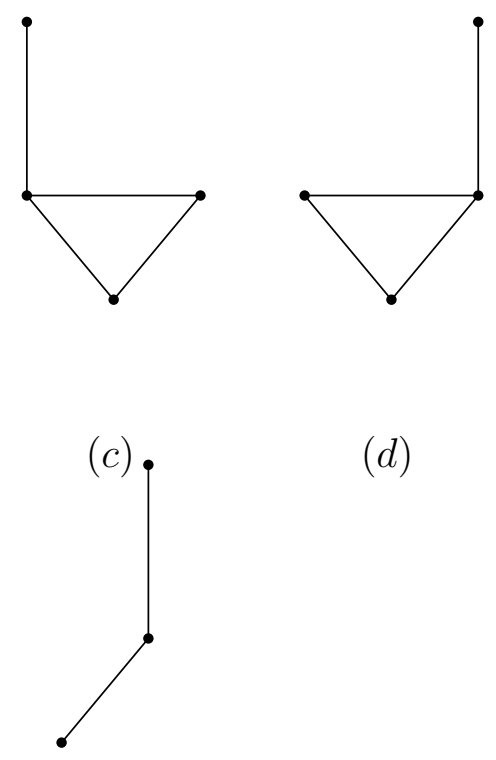

$(d)$

FIGURE 2. (a) A typical two-loop vertex diagram. (a)-(f) Subgraphs contributing to the asymptotic expansion of the diagram (a) in the Sudakov limit.

Note that a typical feature of the off-shell explicit formulae is an interplay between ultraviolet and infrared divergences which appear in individual terms of the expansion but are mutually canceled, provided the initial Feynman integral is finite. It turns out that, for the Sudakov limit, one meets a more general interplay between ultraviolet, collinear and infrared divergences which, at first sight, seem to be of very different nature. In particular, in the case of the Feynman integral (4), which does not have divergences from the very beginning, individual terms of the corresponding asymptotic expansion involve all the three kinds of divergences resulting in poles up to $1 / \epsilon^{4}$, with $\epsilon=(4-$ d) $/ 2$ parameter of dimensional regularization [14]. Namely, the contribution of Fig. 2a (as a subgraph) involves infrared and collinear divergences, the contribution of Fig. 2(e\&f) involves ultraviolet and collinear divergences, while other three contributions, Fig. 2b and Fig. 2(c\&d) possess all kinds of the divergences. However these poles are successfully canceled in the sum and one obtains expansion (5) in the limit $\epsilon \rightarrow 0$.

Note that similar explicit formulae can be derived and applied for other versions of the Sudakov limit, e.g. $p_{i}^{2}=m^{2}$, with $q^{2} \equiv\left(p_{1}-p_{2}\right)^{2} \rightarrow-\infty$. It is natural to expect that the presented possibility to obtain all powers and logarithms in two loops can be applied to extend well-known results on 
asymptotic behaviour in the Sudakov limit in QED and QCD [15].

Extensions of the presented methods and results to other typically Minkowskian limits and their applications will be reported elsewhere.

My participation in the Balholm Workshop has been supported by the Organizing Committee of the workshop and by the Russia Ministry for Science and Technologies. The research has been supported by the Russian Foundation for Basic Research, project 96-01-00654, and by INTAS, project 93-0744.

Acknowledgments. I am grateful to P. Osland for kind hospitality during the workshop and to K.G. Chetyrkin, A. Czarnecki, A.I. Davydychev, K. Melnikov and J.B. Tausk for helpful discussions.

\section{REFERENCES}

1. Gorishny, S.G., Nucl. Phys. B319, 633 (1989).

2. Chetyrkin, K.G., Teor. Mat. Fiz. 75, 26; 76, 207 (1988); preprint MPIPAE/PTh 13/91 (Munich, 1991).

3. Smirnov, V.A., Commun. Math. Phys. 134, 109 (1990); Renormalization and asymptotic expansions, Basel: Birkhäuser, 1991.

4. Smirnov, V.A., Mod. Phys. Lett. A 10, 1485 (1995).

5. Larin, S.A., van Ritbergen, T., and Vermaseren, J.A.M., Nucl. Phys. B438, 278 (1995).

6. Zimmermann, W., Commun. Math. Phys. 15, 208 (1969); Ann. Phys. 77, 570 (1973).

7. Anikin, S.A., and Zavialov, O.I., Teor. Mat. Fiz. 27, 425 (1976); Ann. Phys. 116, 135 (1978); Zavialov, O.I., Renormalized Quantum Field Theory, Kluwer Academic Publishers, 1990.

8. Smirnov, V.A., Phys. Lett. B394, 205 (1997).

9. Czarnecki, A., and Smirnov, V.A., Phys. Lett. B394, 211 (1997).

10. Avdeev, L.V., and Kalmykov, M.Yu., hep-ph/9701308, to appear in Nucl. Phys B.

11. Czarnecki, A., and Melnikov, K., Phys. Rev. Lett. 78, 3630 (1997); hepph/9706227; Czarnecki, A., Melnikov, K., and Uraltsev, N., hep-ph/9706311.

12. Smirnov, V.A., hep-ph $/ 9703357$, to appear in Phys. Lett. B.

13. Chetyrkin, K.G., and Tkachov, F.V., Nucl. Phys. B192, 159 (1981).

14. 't Hooft, G., and Veltman, M., Nucl. Phys. B44, 189 (1972); Bollini, C.G., and Giambiagi, J.J., Nuovo Cim. 12B, 20 (1972).

15. Sudakov, V.V., Zh. Eksp. Teor. Fiz. 30, 87 (1956); Cornwall, J.M., and Tiktopoulos, G., Phys. Rev. D13, 3370 (1976); Belokurov, V.V., and Ussyukina, N.I., Teor. Mat. Fiz. 47, 157 (1979); 44, 147 (1980); Collins, J.C., in Perturbative QCD, ed. Mueller, A.H., 1989, p. 573; Korchemsky, G.P., Phys. Lett. B217, 330 (1989); B220, 629 (1989). 